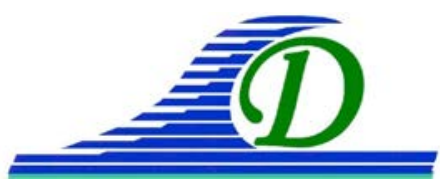
XIII $^{\text {ìmes }}$ Journées Nationales Génie Côtier - Génie Civil Dunkerque, 2-4 juillet 2014

DOI:10.5150/jngcgc.2014.101 @ Editions Paralia CFL

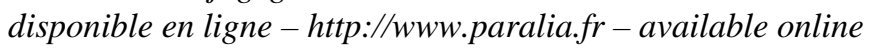

\title{
Vulnérabilité du territoire national à la submersion marine
}

\section{Céline PERHERIN ${ }^{1}$, Amélie ROCHE ${ }^{1}$, Céline TRMAL ${ }^{2}$, Isabelle ROUX ${ }^{2}$, Frédéric PONS $^{2}$, Céline BOURA ${ }^{3}$, Emmanuel DEVAUX $^{3}$, Guy DESIRE $^{3}$}

1. Centre d’Études et d'expertise sur les Risques, l'Environnement, la Mobilité et l'Aménagement (CEREMA/DTecEMF/DI/IE/IAR),

Technopôle brest iroise - 155, rue Pierre Bouguer - BP 5 - 29280 Plouzane, France.

Celine.Perherin@cerema.fr ; Amelie.Roche@cerema.fr

2. Centre d'Etudes et d'expertise sur les Risques, l'Environnement, la Mobilité et l'Aménagement (CEREMA/DTerMed/DREC/SRILH), CS 70499- 13593 Aix-En-Provence Cedex 3, France.

Celine.Trmal@cerema.fr ; Isabelle.Roux@cerema.fr ; Frederic.Pons@cerema.fr

3. Centre d'Études et d'expertise sur les Risques, l'Environnement, la Mobilité et l'Aménagement (CEREMA/DTerOuest/DIMER/Environnement) M.A.N. - Rue René Viviani - BP 46223 - 44262 Nantes Cedex 2, France. Celine.Boura@cerema.fr ; Emmanuel.Devaux@cerema.fr ; Guy.Desire@cerema.fr

\section{Résumé :}

"Vulnérabilité du territoire national aux risques littoraux" est une étude menée par le CETMEF et les CETE Méditerranée et CETE de l'Ouest, aujourd’hui regroupés au sein du Cerema. Les principaux objectifs étaient de faire une synthèse des connaissances sur la vulnérabilité aux risques côtiers et d'établir une représentation cartographique des secteurs vulnérables en France. L'étude a porté sur la métropole et sur l'Outre-mer. La détermination de différents indicateurs, basés sur l'analyse de bases de données disponibles au niveau national (arrêtés de déclaration de l'état de catastrophe naturelle, niveaux marins extrêmes, BD Topo® ${ }^{\circledR}$ IGN et enjeux, dont bâti, infrastructures de transport, établissements Seveso et réseau Natura 2000), a permis de quantifier la vulnérabilité à la submersion marine et de réaliser des cartes de synthèse. La cartographie de la vulnérabilité de chaque commune française permet d'identifier certains secteurs très vulnérables, confirmant ce qui était déjà constaté au niveau national, et de pouvoir les comparer. Ressortent ainsi par exemple le littoral de la façade Nord, la Baie de Somme, les Pays de la Loire, les îles de Charente-Maritime et le Languedoc-Roussillon.

Mots-clés : Vulnérabilité, Risques littoraux, Gestion du littoral, Indicateurs cartographiques.

\section{Introduction}

L'étude "Vulnérabilité du territoire national aux risques littoraux" avait pour objectif de disposer d'une synthèse nationale des connaissances actuelles sur les risques littoraux 


\section{Thème 7 - Risques côtiers}

(érosion des côtes basses meubles et des falaises, avancées dunaires, submersion marine, aléas d'origine météorologique - tempêtes et cyclones) et d'évaluer les secteurs les plus susceptibles d'être impactés par l'élévation du niveau marin. On entend par "vulnérabilité du territoire" la conséquence physique des aléas côtiers sur un territoire exposé, c'est-à-dire, pour la submersion marine par exemple, l'inondation des terres par la mer et ses conséquences sur les enjeux.

Les résultats se présentent notamment sous la forme d'indicateurs cartographiques, qui permettent d'établir une représentation des principales zones vulnérables en France.

Le présent travail présente l'analyse menée sur la vulnérabilité à la submersion marine du territoire français, métropole et Outre-mer comprenant les départements d'Outre-mer (DOM), et certaines collectivités d'Outre-mer (COM) et pays d'Outre-mer (POM). L'ensemble des DOM, COM et POM traités est appelé abusivement territoires d'Outremer dans la suite du document.

\section{Indicateur Zones basses}

La cartographie des zones basses, zones topographiques situées sous un niveau marin de référence, est un indicateur de la vulnérabilité du territoire aux submersions marines. La méthodologie de cartographie reprend celle de l'aléa submersion marine du guide méthodologique "Plans de Prévention des Risques Littoraux" (MATE \& METL, 1997) : on "superpose la cote du plan d'eau retenu à la topographie, pour cartographier les espaces continentaux situés à une altitude inférieure à la cote de référence". Les zones basses obtenues sont distinctes des zones inondables. L'analyse a été conduite sur l'ensemble des territoires où un MNT était disponible.

Le niveau marin de référence retenu est un niveau marin centennal pour les côtes métropolitaines. Sur les côtes Atlantique - Manche - Mer du Nord de France métropolitaine, le niveau marin centennal est extrait de l'étude SHOM-CETMEF (SIMON, 2008). En Méditerranée, la cote de référence a été choisie à partir de la bibliographie et des possibilités offertes par le croisement du MNT et des niveaux marins. En effet, en cohérence avec la précision du MNT, seules les iso-valeurs de niveaux marins correspondant à des demi-valeurs entières (de +2,5 à +8,5 m NGF) ont été retenues. 28 classes ont ainsi été définies sur le littoral métropolitain. La détermination des niveaux de référence en Outre-mer a fait l'objet d'une attention particulière. En effet, la problématique des surcotes cycloniques et les faibles périodes de mesures marégraphiques permettaient difficilement de déterminer statistiquement des niveaux de différentes périodes de retour. Une analyse de la bibliographie existante, et particulièrement des résultats des simulations de surcotes cycloniques réalisées par Météo-France, a permis de proposer des niveaux de référence.

Le traitement du Modèle Numérique de Terrain (MNT) de la BD Topo® de l'IGN, ou d'une BD Topo spécifique pour la Nouvelle-Calédonie dont la densité de points est supérieure, a permis la création de polylignes d'iso-valeurs (valeurs semi-entières) et 


\section{XIII ${ }^{\text {èmes }}$ Journées Nationales Génie Côtier - Génie Civil \\ Dunkerque, 2-4 juillet 2014}

l'obtention de polygones pour le calcul des surfaces. Les limites des zones des différents niveaux marins ont été prolongées dans les terres perpendiculairement au trait de côte Histolitt (IGN-SHOM), sauf en Nouvelle-Calédonie où un trait de côte spécifique a été utilisé.

Les zones basses ainsi déterminées présentent donc plusieurs limites issues de leur propre définition (distinctes des zones inondables par submersion marine) et de la précision des données topographiques utilisées $( \pm 1 \mathrm{~m})$. Les évolutions de la connaissance des niveaux marins retenus pour la détermination des zones basses restent d'un ordre de grandeur bien inférieur à la précision des données topographiques. L'utilisation de données de type LIDAR serait donc susceptible de fortement améliorer la détermination de ces zones.

Ainsi, compte tenu de l'incertitude altimétrique du MNT, et pour étudier le possible impact d'élévation du niveau marin moyen lié au changement climatique (élévation à échéance 100 ans ici retenue : +1 m), trois types de zones ont été étudiées : les zones situées sous le niveau marin de référence, sous le niveau marin de référence moins 1 mètre et sous le niveau marin de référence plus 1 mètre, estimant ainsi les variations de surface en fonction des niveaux marins retenus. Compte tenu des incertitudes supplémentaires sur les niveaux marins de référence retenus en Outre-mer, les zones situées sous le niveau marin de référence plus 2 mètres et moins 2 mètres ont aussi été cartographiées. Les zones en eau, lacs, étangs, cours d'eau, ont été extraites des zones basses à partir de la couche des surfaces en eau de la BD Topo®.

D'après les estimations effectuées (voir résultats tableau 1), en France, 753400 hectares, dont 590000 en métropole en 163100 en Outre-mer, sont situés sous les niveaux marins de référence et 972300 hectares, dont 735500 en métropole et 236800 en Outre-mer, sont situés sous les niveaux marins de référence $+1 \mathrm{~m}$. Les régions dont les surfaces de zones basses sont les plus importantes sont la Guyane, les Pays de la Loire, le PoitouCharentes et le Nord-Pas de Calais. Les régions Guyane, PACA, Aquitaine et Languedoc-Roussillon sont celles dont les surfaces de zones basses augmentent le plus avec une élévation du niveau marin. De manière générale, les zones basses des régions d'Outre-mer et de Méditerranée continentale (Languedoc-Roussillon et PACA) sont très sensibles à une élévation du niveau moyen de la mer puisqu'elles augmentent de plus de $30 \%$ en surface. L'analyse n'a cependant pas pu être conduite à Wallis-et-Futuna ou encore en Polynésie Françaises, traitées par ailleurs dans le cadre de cette étude, faute de disponibilité d'un MNT.

\section{Arrêtés de reconnaissance de l'état de catastrophe naturelle liés à la mer}

La base de données GASPAR (Gestion Assistée des Procédures Administratives relatives aux Risques naturels et technologiques) recense par région les arrêtés interministériels pris lors d'événements exceptionnels reconnus comme catastrophes naturelles. Ces arrêtés constituent un indicateur d'exposition d'une commune à un aléa, 


\section{Thème 7 - Risques côtiers}

qui peut être littoral. L'exploitation de cette base de données a permis d'évaluer et de cartographier le nombre d'arrêtés par commune liés à des phénomènes marins. Un arrêté peut cependant être pris pour un seul bien comme pour un grand nombre de biens touchés.

Tableau 1. Surfaces en hectares des zones basses situées sous les niveaux marins de référence, sous les niveaux marins de référence $-1 m$ et $-2 m$, sous les niveaux marins de référence $+1 \mathrm{~m}$ et $+2 \mathrm{~m}$ et pourcentage des zones basses sous les niveaux marins de référence par rapport à la surface totale du secteur considéré

\begin{tabular}{|c|c|c|c|c|c|}
\hline $\begin{array}{l}\text { Régions métropolitaines, } \\
\text { territoires d'Outre-mer }\end{array}$ & $\begin{array}{l}\text { Niveaux de } \\
\text { référence -2 m }\end{array}$ & $\begin{array}{l}\text { Niveaux de } \\
\text { référence -1 m }\end{array}$ & $\begin{array}{l}\text { Niveaux de référence } \\
\text { (en ha) (\% surface } \\
\text { totale région) }\end{array}$ & $\begin{array}{l}\text { Niveaux de } \\
\text { référence } \\
+1 \mathrm{~m}\end{array}$ & $\begin{array}{l}\text { Niveaux de } \\
\text { référence } \\
+2 \mathrm{~m}\end{array}$ \\
\hline Nord-Pas de Calais & & 72352 & $81674(6,6)$ & 86892 & \\
\hline Picardie & & 19116 & $20458(1,1)$ & 21930 & \\
\hline Haute-Normandie & & 15572 & $25749(2,1)$ & 29008 & \\
\hline Basse-Normandie & & 24864 & $45447(2,6)$ & 59989 & \\
\hline Bretagne & & 25278 & $35614(1,3)$ & 46149 & \\
\hline Pays de la Loire & & 121880 & $163168(5,1)$ & 182950 & \\
\hline Poitou-Charentes & & 54689 & $96244(3,7)$ & 111387 & \\
\hline Aquitaine & & 33908 & $59053(1,4)$ & 82650 & \\
\hline Languedoc-Roussillon & & 2686 & $21782(0,8)$ & 48983 & \\
\hline$P A C A$ & & 4652 & $37915(1,2)$ & 60206 & \\
\hline Corse & & 1761 & $3229(0,4)$ & 5445 & \\
\hline Total Métropole & Non calculé & 376757 & 590331 & 735590 & Non calculé \\
\hline Guadeloupe & 378 & 1766 & $3480(2,1)$ & 5709 & 7818 \\
\hline Martinique & 50 & 331 & $1192(1,1)$ & 2333 & 3571 \\
\hline Guyane & 13812 & 65019 & $124157(1,5)$ & 178045 & 249125 \\
\hline Réunion & 16 & 316 & $696(0,3)$ & 1150 & 1765 \\
\hline Mayotte & 152 & 264 & $441(1,2)$ & 684 & 951 \\
\hline St Martin & 26 & 121 & $230(2,5)$ & 321 & 472 \\
\hline St Barthélémy & 22 & 56 & $88(3,5)$ & 121 & 158 \\
\hline St Pierre et Miquelon & 55 & 359 & $672(2,8)$ & 985 & 1266 \\
\hline Nouvelle-Calédonie & 13579 & 24118 & $36124(1,9)$ & 47412 & 60406 \\
\hline Total Outre-mer & 28080 & 92350 & 163080 & 236760 & 325532 \\
\hline Total France & Non calculé & 469107 & 753411 & 972350 & Non calculé \\
\hline
\end{tabular}

La terminologie des risques inscrits dans la base a été traitée car les intitulés ne sont pas directement liés à l'action de la mer, l'analyse ayant porté sur les arrêtés concernant les communes situées dans les zones basses définies ci-dessus.

Le résultat est un nombre de catastrophes naturelles par commune. Les résultats (voir figure 1) ne sont pas valables au niveau local mais national, les intitulés étant attribués de façon assez libre. La représentation des communes ayant le plus grand nombre d'arrêtés tend vers des couleurs rouges/foncées. Les régions Bretagne et BasseNormandie concentrent les communes ayant les plus grands nombres d'arrêtés. Les Alpes-Maritimes ressortent aussi, ainsi que les estuaires (Gironde et Seine), la Réunion, la Guadeloupe et la Martinique. Ces 3 DOM sont loin derrière les deux régions comptabilisant les plus grands nombres d'arrêtés, Bretagne et Basse-Normandie, ainsi 


\section{XIII ${ }^{\text {èmes }}$ Journées Nationales Génie Côtier - Génie Civil \\ Dunkerque, 2-4 juillet 2014}

que du Nord-Pas de Calais, de la Haute-Normandie, des Pays de la Loire, du PoitouCharentes ou de l'Aquitaine, mais cette information est à relativiser au regard de la surface des communes, plus importante en Outre-mer. Le pourcentage de communes d'une région disposant d'un grand nombre d'arrêtés fait ressortir la Martinique. Les arrêtés couvrent l'ensemble des communes littorales des départements, sauf la Corse et le Var.

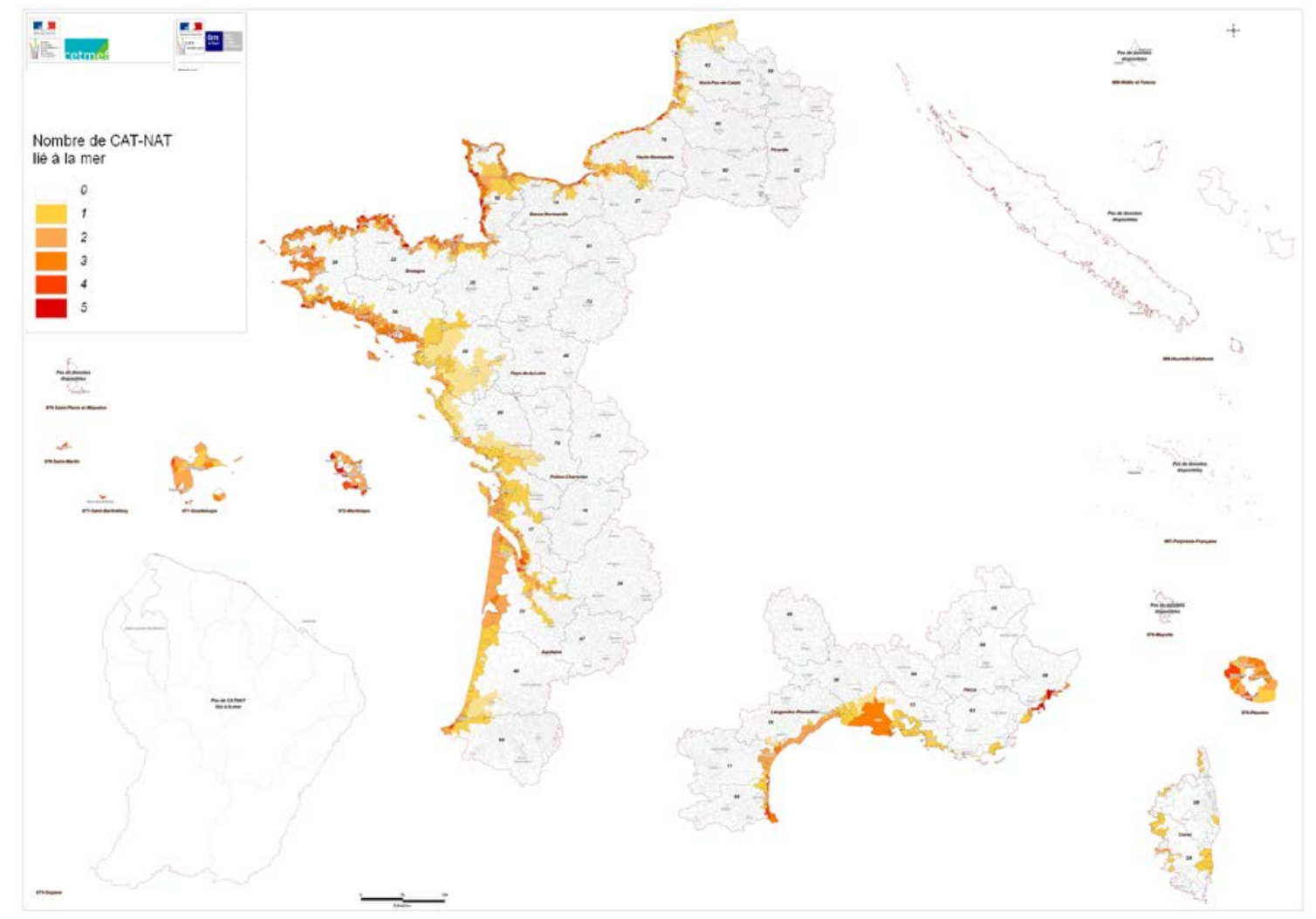

Figure 1. Carte des arrêtés de catastrophe naturelle liés à la mer.

\section{Enjeux situés dans les zones basses}

Un indicateur de vulnérabilité basé sur un certain nombre d'enjeux, pour lesquels des bases de données étaient disponibles à l'échelle nationale, a été défini. Ces enjeux ont été comptabilisés dans les zones basses préalablement définies, rendant ainsi compte d'un degré de vulnérabilité à la submersion marine. Il s'agit: des constructions (bâtiments administratifs, industriels, résidentiels...) et des infrastructures de transports (voies routières et ferroviaires) issues de la BD Topo Pays ${ }^{\circledR}$ de l'IGN, des établissements industriels à risques (classés SEVESO et centrales nucléaires) et des sites d'intérêt écologique (Natura 2000, ZNIEFF, réserves et parc...). L'ensemble de ces enjeux a été déterminé pour chaque type de zones basses (sous les niveaux marins de référence, voir tableau 2, et sous les niveaux marins de référence $-1 \mathrm{~m}$ et $+1 \mathrm{~m}$ ) dans 


\section{Thème 7 - Risques côtiers}

chaque région littorale de métropole, ainsi que sur chacun des territoires d'Outre-mer pris en compte par l'étude.

Les données analysées diffèrent parfois légèrement (structure des données, type de protections réglementaires existantes...) entre la métropole et l'Outre-mer. Des vérifications à partir d'ortho-photographies ont également permis de s'assurer que les bâtis légers étaient pris en compte dans la base de données. Les quelques modifications constatées sont cependant compatibles à l'échelle et aux objectifs de l'analyse. Les fortes évolutions constatées sur les bases de données relatives aux enjeux mises à disposition par l'INSEE ou l'IGN (carroyage INSEE mis à disposition depuis 2013 notamment) permettraient aujourd’hui des analyses améliorées.

Tableau 2. Enjeux situés dans les zones basses sous les niveaux marins de référence.

\begin{tabular}{|c|c|c|c|}
\hline $\begin{array}{l}\text { Régions métropolitaines, } \\
\text { territoires d'Outre-mer }\end{array}$ & $\begin{array}{l}\text { Nombre de } \\
\text { bâtiments }\end{array}$ & $\begin{array}{l}\text { Linéaire } \\
\text { d'infrastructures } \\
\text { de transport }(\mathrm{km})\end{array}$ & $\begin{array}{l}\text { Surface de sites } \\
\text { d'intérêt } \\
\text { écologique (ha) }\end{array}$ \\
\hline Nord-Pas de Calais & 65676 & 3757 & 1918 \\
\hline Picardie & 10149 & 545 & 5445 \\
\hline Haute-Normandie & 9003 & 923 & 12410 \\
\hline Basse-Normandie & 15591 & 868 & 28923 \\
\hline Bretagne & 22375 & 1611 & 15540 \\
\hline Pays de la Loire & 59658 & 3074 & 125901 \\
\hline Poitou-Charentes & 28762 & 1783 & 64052 \\
\hline Aquitaine & 25916 & 1582 & 34367 \\
\hline Languedoc-Roussillon & 16135 & 1905 & 27752 \\
\hline PACA & 10571 & 711 & 64973 \\
\hline Corse & 1320 & 45 & 1359 \\
\hline Total Métropole & 265156 & 16804 & 382640 \\
\hline Guadeloupe & 5161 & 262 & 2432 \\
\hline Martinique & 2487 & 103 & 19 \\
\hline Guyane & 3089 & 371 & 25910 \\
\hline Réunion & 1338 & 78 & 68 \\
\hline Mayotte & 1353 & 33 & 0 \\
\hline St Martin & 1012 & 33 & 10 \\
\hline St Barthélémy & 197 & 7 & 9 \\
\hline St Pierre et Miquelon & 472 & 72 & 0 \\
\hline Nouvelle-Calédonie & 9486 & 304 & 19 \\
\hline Total Outre-mer & 24595 & 1263 & 28448 \\
\hline
\end{tabular}

En métropole, les constructions et les infrastructures de transports concernent principalement les régions dont les surfaces de zones basses sont les plus élevées : le Nord-Pas de Calais, les Pays de la Loire, le Poitou-Charentes, le Languedoc-Roussillon et l'Aquitaine. La concentration des enjeux bâtis dans les zones basses est particulièrement importante en Outre-mer, en particulier, par ordre d'importance, St Martin, Mayotte, St Barthélémy, la Martinique, la Réunion et la Guadeloupe, tous devant les régions de métropole. Les établissements industriels à risques des communes littorales sont essentiellement situés dans les zones portuaires (Grands Ports Maritimes de Marseille, Le Havre, Rouen et Dunkerque). Les zonages de protections 


\section{XIII ${ }^{\text {èmes }}$ Journées Nationales Génie Côtier - Génie Civil \\ Dunkerque, 2-4 juillet 2014}

environnementales représentent près de 411000 ha et $54 \%$ des surfaces situées sous les niveaux marins de référence (383000 ha et $64 \%$ pour la métropole), concernant essentiellement les Pays de la Loire, Poitou-Charentes et PACA.

\section{Conclusions : Indicateurs croisés}

Un indicateur seul ne donne qu'une image partielle de la vulnérabilité ; le croisement des différents indicateurs liés aux aléas et aux enjeux est un élément de réponse pour cartographier la vulnérabilité à la submersion marine. Il a été choisi de croiser les arrêtés de catastrophes naturelles et les bâtiments situés dans les zones basses par commune, en considérant qu'une commune sera plus vulnérable si elle contient un grand nombre de bâtiments et qu'elle a subi de nombreuses catastrophes naturelles liées à la mer. Dans cet indicateur, appelé IBC, six niveaux de vulnérabilité ont été définis. Parmi les territoires traités, les régions les plus vulnérables sont le Languedoc-Roussillon, la Picardie, le Poitou-Charentes, le Nord-Pas de Calais et les Pays de la Loire, au contraire des régions Corse, Haute-Normandie, Basse-Normandie et Bretagne. D’après cette analyse, les territoires d'Outre-mer apparaissent comme peu vulnérables en dehors de la commune de St Denis à la Réunion.

L'absence d'arrêté CAT-NAT dans plusieurs territoires d'Outre-mer a conduit à la recherche d'un nouvel indicateur afin d'avoir une représentation plus précise de la vulnérabilité à la submersion marine à l'échelle de la France. Les données disponibles ont permis la réalisation d'un indicateur correspondant au nombre de bâtiments dans les zones basses d'une commune, indicateur appelé IB (voir figure 2) présentant également 6 niveaux de vulnérabilité, les niveaux les plus forts tendant vers le rouge/foncé. Sur les régions où les indicateurs IB et IBC ont été cartographiés, les variations de la vulnérabilité d'une commune à une autre restent globalement identiques.

La plupart des régions font apparaître des communes vulnérables de manière isolée (St Malo, Penmarc'h, Carnac en Bretagne, La Cap Ferret, Bordeaux, le Verdon en Aquitaine, Hyères en PACA, Le Havre en Haute-Normandie, ...) ou sur des secteurs plus étendus (la façade Mer du Nord, la Baie de Somme, le nord de l'embouchure de la Loire, la Vendée, les îles de Charente-Maritime, le littoral de l'Aude et de manière plus générale le Languedoc-Roussillon, la Camargue, le sud de la Nouvelle-Calédonie). La cartographie a aussi été réalisée pour le bâti sous les niveaux marins de référence $+1 \mathrm{~m}$ afin d'évaluer l'évolution de la vulnérabilité avec l'élévation du niveau moyen de la mer. En métropole, la région où l'évolution est la plus forte est le Languedoc-Roussillon. L'Outre-mer y est également vulnérable, en particulier la Martinique, suivie de la Réunion et de la Guyane. 


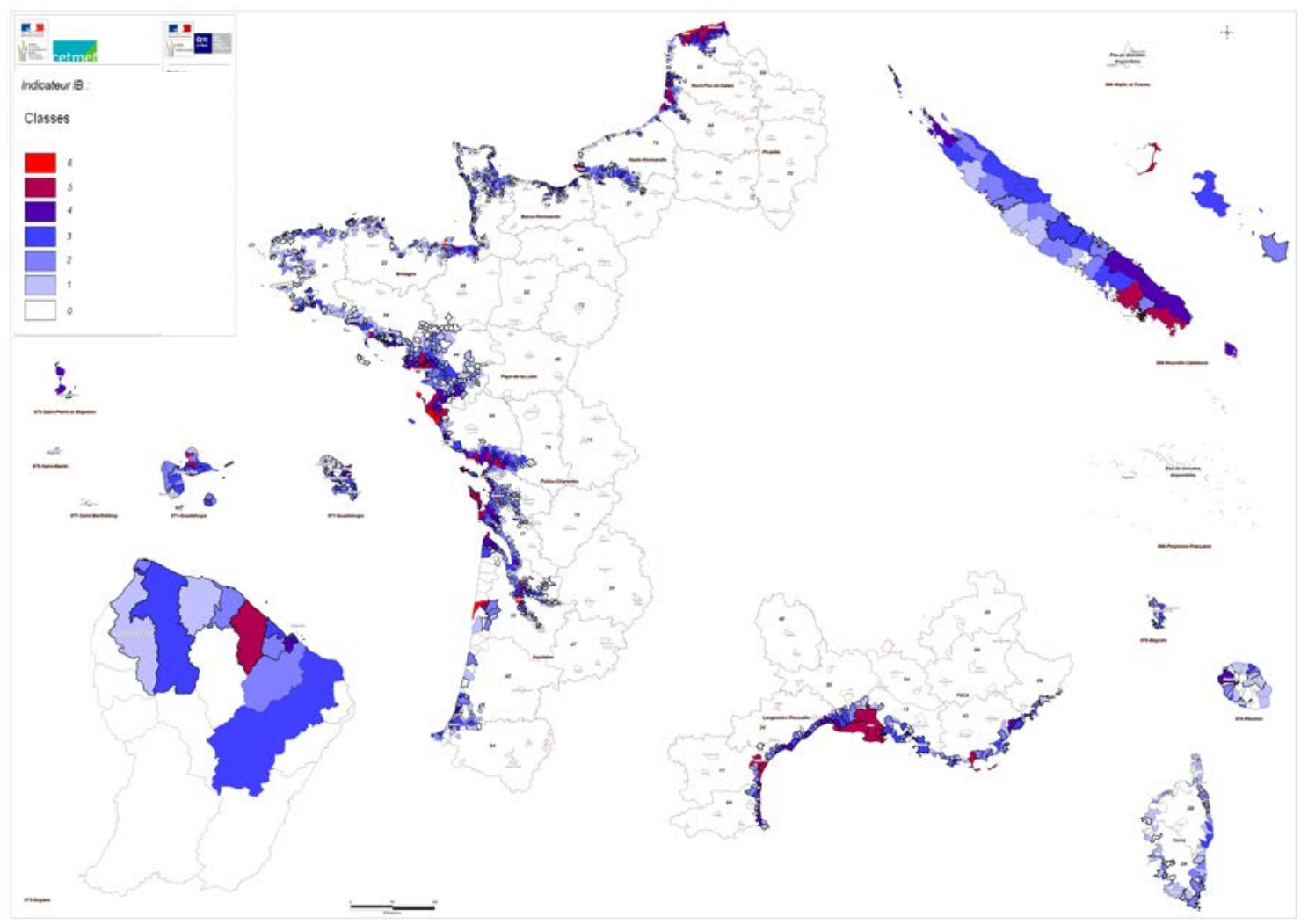

Figure 2. Carte de l'indicateur croisé de vulnérabilité aux risques littoraux (IB).

\section{Références bibliographiques}

CETMEF, CETE Méditerranée, CETE Ouest (2012). Vulnérabilité du territoire national aux risques littoraux - France métropolitaine. Rapport CETMEF, Référence CETMEF : C 12.03, 170 p.

CETMEF, CETE Méditerranée, CETE Ouest (2012). Vulnérabilité du territoire National aux risques littoraux. - Outre-mer. Rapport CETMEF, Référence CETMEF : C 12.03, $160 \mathrm{p}$.

CETMEF, CETE Méditerranée, CETE Ouest (2012). Vulnérabilité du territoire National aux risques littoraux. - France entière. Rapport CETMEF, Référence CETMEF : C 12.03, 52 p.

MATE, METL -Ministère de l'Aménagement du Territoire et de l'Environnement, Ministère de l'Équipement, des Transports et du Logement- (1997). Plan de prévention des risques littoraux (PPR). Guide méthodologique. La documentation française, Paris, $54 \mathrm{p}$.

SIMON B. (2008). Statistiques des niveaux marins extrêmes de pleine mer Manche et Atlantique. SHOM-CETMEF, Cédérom. 